\title{
Femtosecond laser-induced forward transfer of thin layers studied by time-resolved shadowgraphy
}

\author{
M. Feinaeugle ${ }^{1}$, A. P. Alloncle ${ }^{2}$, Ph. Delaporte ${ }^{2}$, C. L. Sones ${ }^{1}$, R. W. Eason ${ }^{1}$ \\ ${ }^{1}$ Optoelectronics Research Centre, University of Southampton, Southampton, SO17 1BJ, U.K. \\ ${ }^{2}$ Laboratoire LP3 (Lasers, Plasma et Procédés Photoniques), UMR 6182 CNRS, Université de la Méditerranée, Campus de Luminy C917, 13288 \\ Marseille Cedex 09, France \\ Correspondent author e-mail address (M. Feinaeugle): mf2v09@orc.soton.ac.uk, Tel: (+44) 0238059 9091, Fax: (+44) 02380593142
}

\begin{abstract}
We report the shadowgraph imaging of femtosecond laser-induced forward transfer of $0.5-1.8 \mu \mathrm{m}$ solid films. We observe intact transfer with velocities as low as $34 \mathrm{~m} / \mathrm{s}$ in the absence of any shock wave.

OCIS codes: (350.3390) Laser materials processing; (320.2250) Femtosecond phenomena
\end{abstract}

\section{Introduction}

Laser-induced forward transfer (LIFT) is a laser-assisted direct-write method that has been explored for the fabrication of electronic and photonic devices [1]. LIFT using a laser source with femtosecond pulses (fs-LIFT) has also been investigated since the use of a fs-laser was expected to reduce the interfacial thermal damage of the material to be transferred, when compared to LIFT employing picosecond (ps) or nanosecond (ns) pulses. In order to further establish the feasibility of LIFT as a valuable micro-fabrication tool, it is of high importance to understand and investigate the dynamic processes involved during the transfer of a thin film (the donor) onto a receiving substrate (the receiver). In this contribution, we studied fs-LIFT of solid films via a time-resolved shadowgraphy technique with an aim of identifying the velocity of the transferred product, the behaviour and integrity of the donor in transfer (the flyer), and the role of the creation of shock waves.

\section{Methods}

The LIFT setup used was based on a 100fs-Ti:Sapphire laser system (Spectra-Physics Hurricane) described in [2] and shown in Fig. 1. Single laser pulses were imaged onto the interface between the transparent carrier and the donor resulting in an illuminated area of $\sim 25 \mu \mathrm{m}$ in diameter. A collimated beam from a flash lamp (HSPS Nanolite) incoherently illuminated the volume above the donor to shadowgraphically image objects and atmospheric distortions with a gated CCD camera. A delay generator synchronized the flash lamp with the camera in order to capture events above the donor at specific times of up to $10 \mu \mathrm{s}$ after the arrival of the fs pulse at the donor. Donor films of $1.1 \mu \mathrm{m}$ thick bismuth selenide $\left(\mathrm{Bi}_{2} \mathrm{Se}_{3}\right), 1.8 \mu \mathrm{m}$ thick lead zirconate titanate (PZT) and Terfenol-D with a thickness of $0.5 \mu \mathrm{m}$ were initially prepared by sputtering onto $2 \mathrm{~mm}$ thick quartz carriers prior to the LIFT experiments.

\section{Results and discussion}

The threshold fluence for the fs-LIFT-transfer of $\mathrm{Bi}_{2} \mathrm{Se}_{3}$ was observed to be $\sim 90 \pm 10 \mathrm{~mJ} / \mathrm{cm}^{2}$, and the ejected flyers remained intact in about $50 \%$ of the cases when the fluence was around this threshold. The flyers fragmented in the other cases. The measured velocity of the flyer for times larger than $1 \mu$ s was $\sim 48 \pm 7 \mathrm{~m} / \mathrm{s}$. Similarly, for PZT an intact flyer that subsequently travelled with a velocity of $34 \pm 5 \mathrm{~m} / \mathrm{s}$ was released for a threshold fluence of $\sim 360 \mathrm{~mJ} / \mathrm{cm}^{2}$. In case of both the above mentioned donors, increasing fluences resulted in the ejection of a fragmented flyer. For a fluence of $\sim 720 \mathrm{~mJ} / \mathrm{cm}^{2}$ the fastest particles within a fragmented PZT flyer was seen to travel with a velocity of $\sim 360 \pm 15 \mathrm{~m} / \mathrm{s}$. Likewise, a velocity of $354 \pm 12 \mathrm{~m} / \mathrm{s}$, exceeding the speed of sound in air $(331.3 \mathrm{~m} / \mathrm{s})$, was seen for a fragmented $\mathrm{Bi}_{2} \mathrm{Se}_{3}$ flyer for a fluence of $\sim 400 \mathrm{~mJ} / \mathrm{cm}^{2}$. Fig. 1 shows the image sequence of the transfer of an intact (Fig. 1a) and a fragmented (Fig. 1b) flyer of $\mathrm{Bi}_{2} \mathrm{Se}_{3}$ for a fluence of $\sim 130 \mathrm{~mJ} / \mathrm{cm}^{2}$ and $\sim 400 \mathrm{~mJ} / \mathrm{cm}^{2}$ respectively. The fs-LIFT transfer of the Terfenol-D donor almost always resulted in a transfer product with a non-intact structure. The transfer appeared to be similar to LIFT of a rheological fluid [3], hence we assume that the ejected particle had undergone complete melting by the absorbed laser beam.

During all our LIFT experiments, the shadowgrams captured did not show any trace of a shock wave lagging or preceding the ejected species of intact or fragmented flyer. In our study of the fs-LIFT process by time-resolved shadowgraphy we observed that flyers travelled with a velocity lower than those reported before in other LIFT experiments. During ns-LIFT with the use of an auxiliary absorption layer (dynamic release layer or DRL), flyers 
with thickness comparable to those reported here were transferred with a similar fluence but at a higher ejection velocity [4].

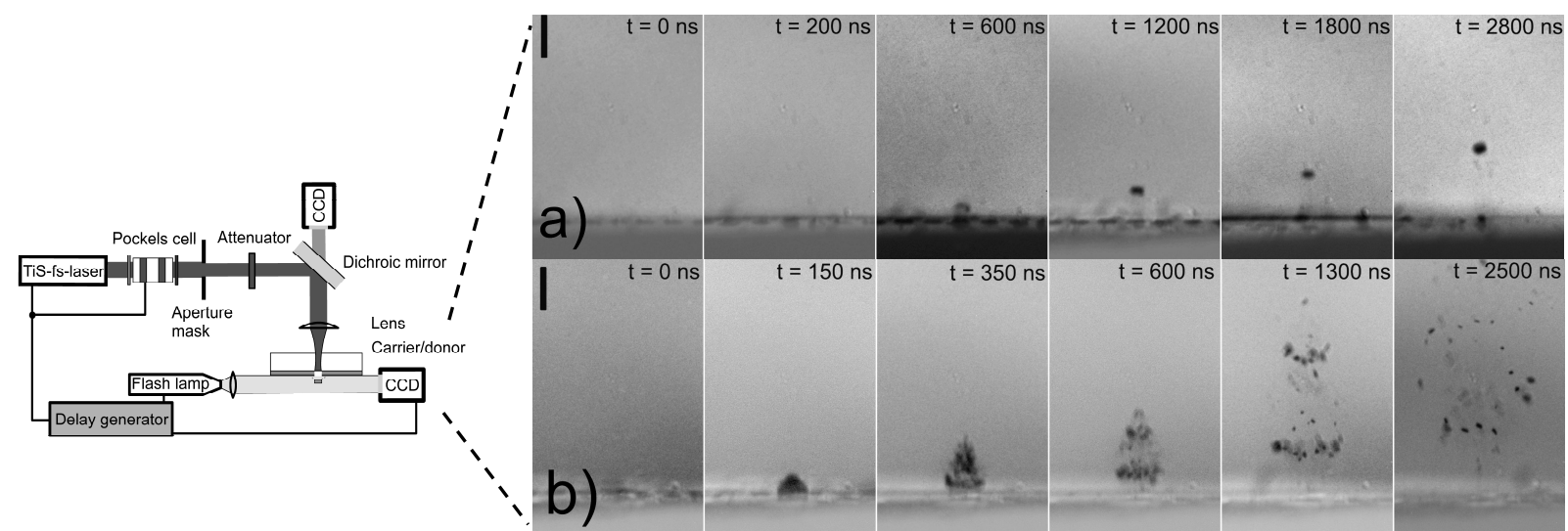

Fig. 1: Schematic of the fs-LIFT shadowgraphy setup. Sequence of shadowgrams showing transfer of a $\mathrm{Bi}_{2} \mathrm{Se}_{3}$ for a fluence of (1a) $\sim 130 \mathrm{~mJ} / \mathrm{cm} 2$ and $(1 \mathrm{~b}) \sim 400 \mathrm{~mJ} / \mathrm{cm} 2$. The times at the bottom of the frames indicate the delay times between arrival of the laser pulse and the beginning of image capture by the CCD. The scale bar in the first frames for a delay time of 0ns in each row is $100 \mu \mathrm{m}$.

In another work, where the author used a modified LIFT technique for the transfer of electronic dies from a support substrate, transfer velocities were found to be as low as $\sim 0.1-10 \mathrm{~m} / \mathrm{s}$ with thicker flyers than used here [5]. Another fs-LIFT experiment [2] reports a velocity of $\sim 50 \mathrm{~m} / \mathrm{s}$ for ejected species of a $40 \mathrm{~nm}$ donor layer. The laser fluence, donor thickness and the use of a DRL appears to have a major influence on the velocity of the transferring flyer. During our experiments, contrary to what has been observed before in LIFT with [4,6] and without [7] the use of a DRL, we could not detect a shock wave propagating in the volume above the donor substrate. A shock wave that is generated during the transfer, when reflected from a receiver placed nearby [6], has the undesired influence of destructively interacting with the flyer. We believe that the absence of a shock wave may be attributed either to the specific properties of the donors, their resultant low flyer velocity, or to the specific transfer/ablation behavior of the fs pulses. The low flyer velocity we have observed for the layers of $\mathrm{Bi}_{2} \mathrm{Se}_{3}$ and PZT may be credited to the relatively large donor thickness of $\sim 1.1-1.8 \mu \mathrm{m}$.

\section{Summary}

We report the first ever shadowgraphy studies of intact flyers from solid films of $\mathrm{Bi}_{2} \mathrm{Se}_{3}, \mathrm{PZT}$ and Terfenol-D transferred via fs-laser-induced forward transfer. The lowest transfer velocities observed for $\sim 1.1 \mu \mathrm{m} \mathrm{thick} \mathrm{Bi}_{2} \mathrm{Se}_{3}$, $\sim 1.8 \mu \mathrm{m}$ thick PZT and Terfenol-D with a thickness of $\sim 0.5 \mu \mathrm{m}$ were $\sim 34 \mathrm{~m} / \mathrm{s}, \sim 48 \mathrm{~m} / \mathrm{s}$ and $\sim 140 \mathrm{~m} / \mathrm{s}$ respectively, which are, to the best of our knowledge, the lowest velocities observed for an intact flyer in LIFT without incorporation of auxiliary release layers. Furthermore, we could not observe any shock wave propagating in the space above the donor substrate for the measured time delays, and this together with low flyer velocities is advantageous when transferring delicate materials.

\section{References}

[1] C. B. Arnold, P. Serra and A. Pique, "Laser direct-write techniques for printing of complex materials," MRS Bull. 32, 23-31 (2007).

[2] A. P. Alloncle, R. Bouffaron, J. Hermann and M. Sentis, "Experimental study of front and back ablation of metal thin film using ultrashort laser pulses," in High-Power Laser Ablation VI, Pts 1 and 2., Proceedings of the Society of Photo-Optical Instrumentation Engineers (Spie), eds. C. R. Phipps. Vol. 6261, (Spie-Int Soc Optical Engineering, 2006), pp. 26127-26127.

[3] D. Young, R. C. Y. Auyeung, A. Pique, D. B. Chrisey and D. D. Dlott, "Plume and jetting regimes in a laser based forward transfer process as observed by time-resolved optical microscopy," Appl Surf Sci 197-198, 181-187 (2002).

[4] K. S. Kaur, R. Fardel, T. C. May-Smith, M. Nagel, D. P. Banks, C. Grivas, T. Lippert and R. W. Eason, "Shadowgraphic studies of triazene assisted laser-induced forward transfer of ceramic thin films," J Appl Phys 105, 113119-113118 (2009).

[5] N. S. Karlitskaya, J. Meijer, D. F. de Lange and H. Kettelarij, "Laser propulsion of microelectronic components: releasing mechanism investigation," in High-Power Laser Ablation VI, Pts 1 and 2, Proceedings of the Society of Photo-Optical Instrumentation Engineers (Spie), eds. C. R. Phipps, Vol. 6261 (Spie-Int Soc Optical Engineering, 2006), pp. 62612P-1 - 62612P-10.

[6] R. Fardel, M. Nagel, F. Nuesch, T. Lippert and A. Wokaun, "Laser-Induced Forward Transfer of Organic LED Building Blocks Studied by Time-Resolved Shadowgraphy," J Phys Chem C 114, 5617-5636 (2010).

[7] I. Zergioti, D. G. Papazoglou, A. Karaiskou, C. Fotakis, E. Gamaly and A. Rode, "A comparative schlieren imaging study between ns and sub-ps laser forward transfer of Cr," Appl Surf Sci 208-209, 177-180 (2003). 